\title{
Characteristics of Pseudospark Discharge in Particle-in-Cell simulations
}

\author{
Liang Zhang, Wenlong He, Xiaodong Chen, Jin Zhang, and Adrian W. Cross
}

\begin{abstract}
In this paper, the characteristics of the pseudospark (PS) discharge were studied through particle-in-cell simulations with the consideration of the external circuit. The different discharge stages, including the Townsend discharge, hollowcathode discharge and super-dense glow, were characterized by the discharge voltage and current. The sources of electrons at different discharge stages were analyzed, with the electrons generated from the ionization of the background gas playing the dominant role. The electron densities at the Townsend discharge, hollow-cathode discharge and super-dense glow discharge stages were in the range of $10^{13} \mathrm{~m}^{-3}, 10^{18} \mathrm{~m}^{-3}$ and $10^{20} \mathrm{~m}^{-3}$, respectively. The energy of the electron beam extracted at the anode aperture at the different discharge phases was exported and post-processed. The electron beams generated at the three stages have an energy spread of $10 \%, 20 \%$ and $40 \%$, respectively. The hollow-cathode discharge phase has balanced parameters in beam energy, energy spread, and electron density in contrast to the Townsend discharge and super-dense glow discharge stage.
\end{abstract}

Index Terms - pseudospark discharge, self-breakdown, particle-in-cell simulation, low press gas discharge.

\section{INTRODUCTION}

Pseudospark (PS) discharge is a low-pressure discharge process that is characterized by a rapid breakdown phase with the generation of a high current density electron beam [1]. Since its first experimental study in 1978 [2], various research has been carried out to explore its mechanism and applications. The PS discharge process is commonly explained by three stages, including the pre-breakdown (Townsend discharge) phase to ignite the discharge, the hollow-cathode discharge phase with a relatively low discharge current, and the super-dense glow discharge phase with a very high discharge current in the order of few or tens kiloamperes, depending on the geometry of the discharge cavity [3].

The electron beam generated from the PS discharge has high brightness $\left(\sim 10^{12} \mathrm{~A} / \mathrm{m}^{2} \mathrm{rad}^{2}\right)$ and large current density $\left(\sim 10^{4} \mathrm{~A} / \mathrm{cm}^{2}\right)$. It can be focused by the self-magnetic field due to the existence of an ion channel and it can transport a distance of a few tens of centimeters. Therefore it can be used for plasma processing for material treatment [4], extreme-ultraviolet light sources for lithography $[5,6]$, generation of high power millimeter/terahertz wave radiation [7, 8], and so on. At the super-dense glow discharge stage, the beam current can reach tens of kiloamperes in nanoseconds, and it is widely used as

This work was supported by the UK Engineering and Physical Sciences Research Council (EPSRC) under Grant EP/S00968X/1 and EP/S009582/1.

L. Zhang (liang.zhang@strath.ac.uk), and A.W. Cross (a.w.cross@strath. ac.uk) are with the Department of Physics, University of Strathclyde, Glasgow, G4 0NG, UK. J. Zhang (jin.zhang@qmul.ac.uk), and X. Chen (xiaodong.chen @qmul.ac.uk) are with the School of Electronic Engineering and Computer high-power high-current fast switches in pulsed power systems $[9,10]$.

Experiments and simulations have been carried out to study the discharge mechanism in the last few decades [3, 11-13]. In the experiments, the discharge mechanism was analyzed from the data by diagnosing the discharge current and voltage using fast response probes, the generated electron beam using the Faraday cup enables the electron energy to be measured and the plasma density measured using a Langmuir probe. However, the PS discharge is a fast-transient process, it is challenging to capture all the dynamic behavior during the discharge, especially as there is limited space for the probes when developing a smaller-size compact PS discharge cavity. Numerical simulations can provide extra dynamic information on the discharge process, such as the temporal and spatial distribution of the electric field and plasma density, as well as the contribution of the secondary electrons to the discharge current. A 2D hybrid fluid particle and Monte Carlo model was used to study the evolution of the ignition of the PS discharge, where a local field approximation was used to describe the momentum balance [14]. Later particle-in-cell (PIC) simulation was used to take into account the motion of the electrons in the plasma field more accurately [15]. Since then more simulations were carried out to investigate the impacts to the discharge current and breakdown time from different discharge parameters, including the pressure of the background gas and the dimensions of the discharge cavity $[16,17]$.

Most of the PS discharge PIC simulations in the published papers were done by XOOPIC [18] developed by the University of California at Berkeley or its windows version OOPIC Pro [19]. In these simulations, an ideal voltage source was used between the cathode and anode to drive the discharge. Useful results can be obtained at the Townsend discharge phase and the hollow-cathode discharge stage. However, the simulation can suffer instability if the simulated time is long as well as at a large discharge current during the super-dense glow discharge stage. The plasma density would grow indefinitely due to the infinite power that an ideal voltage source can provide. While in a real device, the large external charging resistance and the finite charging capacitor will eliminate such instability. It will be useful to implement the external circuit model in the simulations, to take into account the feedback of the charging circuit and to obtain a better understanding of the discharge process [20]. External circuit models have been implemented in PIC code since 1990s, such as the XPDP2 [21-24] which was

Science, Queen Marry University of London, London, E1 4NS, UK. W. He (wenlong.he@szu.edu.cn) is with the College of Electronic Science and Technology, Shenzhen University, Shenzhen 518060, China.

Data underpinning this publication is available from the University of Strathclyde at https://doi.org/10.15129/f66e53b5-be1e-40b8-a0a7-9de9550dc 8dd. 
used to simulate the capacitive coupled RF gas discharge. The same method can be used although the external circuits for the capacitive coupled RF gas discharge and DC PS discharge are different.

At the Townsend discharge stage, the electric field strength is weak and the field emission can be ignored. As the virtual anode is formed and keeps expanding, the electric field on the cavity wall increases. At the super-dense glow discharge stage, the field emission can happen since the virtual anode is close to the cavity wall. It is useful to know when the field emission electrons are generated and how significant their contribution is to the discharge process.

In this paper, the external charging circuit for the PS discharge experiment was implemented in PIC code XOOPIC and it was used to simulate the PS discharge process. The experimental configuration of the PS discharge, as well as the circuit model, is presented in section II. The simulation model and the results are represented in sections III and IV, respectively and a conclusion is given in section $\mathrm{V}$.

\section{CONFIGURATION OF PS DISCHARGE EXPERIMENT}

A typical PS discharge setup is shown in Fig. 1. A dielectric plate, either ceramic or Perspex material, is placed between the cathode and anode for insulation as well as to seal the gas. The typical breakdown voltage for a single-gap discharge cavity is less than $20 \mathrm{kV}$. A higher breakdown voltage requires a longer single-disk insulator, however it has the problems of flashover and non-uniform electric field, which affect the stability of the PS discharge. More intermediate metal plates can be placed in turn with the insulators to form the multi-gap configuration. It is able to achieve a uniform electric field and to reduce the time jitter when operating in a high repetition rate mode. Since the self-capacitance between the cathode and anode is small. External energy storage capacitors, which are charged to high voltage through a charging resistor, are used to provide the discharge energy. The gas pressure inside the cavity can be adjusted by the needle valve for the gas inlet or the valve connected to the vacuum pump. At a balance status, the cavity will maintain a stable gas pressure. The discharge voltage is measured by a voltage probe. The discharge current can be measured by a Rogowski coil. The shape of the discharge electron beam can be formed by different apertures at the anode. The electron beam current generated from the discharge process can be measured by a Faraday cup which is placed at a distance to the anode. A scintillator disk can also be used to image the shape of the electron beam.

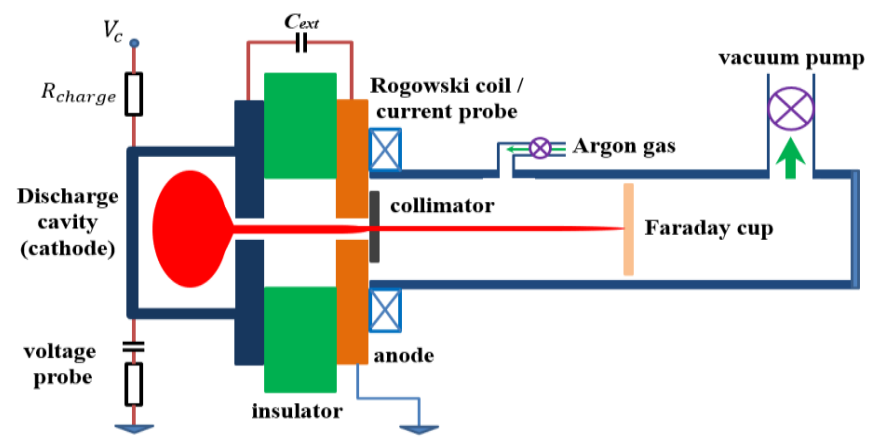

Fig. 1 Schematic drawing of the PS discharge experimental setup.
The discharge process can be described with a circuit model [25, 26], as shown in Fig. 2, where $R_{\text {charge }}$ and $V_{c}$ are charging resistor and voltage applied to the external capacitor $C_{\text {ext }}$. The PS discharge chamber has the self-capacitance and selfinductance of $C_{\text {self }}$ and $L_{\text {self }}$, respectively. $L_{p s}$ and $R_{p s}$ are the inductance and resistance during the discharge due to the oscillation of the plasma. The equations of the discharge voltage and current can be derived from Kirchhoff's law and written as

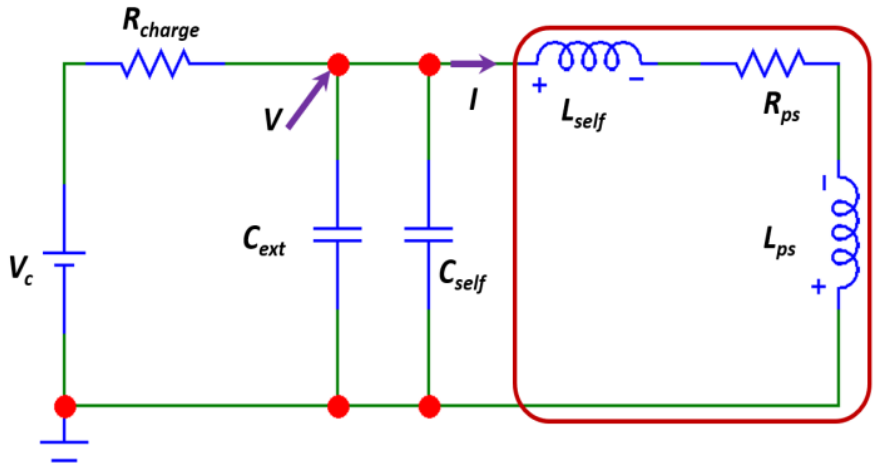

Fig. 2 Circuit model of the discharge process.

$$
\begin{gathered}
\left(C_{\text {ext }}+C_{\text {self }}\right) \frac{d V}{d t}=\left(V_{c}-V\right) / R_{\text {charge }}-I \\
\left(L_{\text {self }}+L_{p s}\right) \frac{d I}{d t}=V-R_{p s} I
\end{gathered}
$$

The RLC discharge equations can be solved as eq. 2 and 3. In the experiment, the $C_{\text {self }}$ and $L_{\text {self }}$ is normally in the order of $40 \mathrm{pF}$ and $100 \mathrm{nH}$. At a small discharge current (corresponding to a large $R_{p s}$ ), for example during the Townsend discharge stage, the voltage will not be damped as $\omega^{2}<0$. While at the super-dense glow discharge stage, the oscillation frequency in the discharge voltage will mostly be determined by the first term of equ. 3 since $R_{p s}$ is a small value.

$$
\begin{gathered}
V(t)=V_{c} \exp \left(-\frac{R_{p s} t}{2\left(L_{\text {self }}+L_{p s}\right)}\right) \cos (\omega t) \\
I(t)=V_{C} \sqrt{\frac{C_{\text {ext }}+C_{\text {self }}}{L_{\text {self }}+L_{p s}}} \exp \left(-\frac{R_{p s} t}{2\left(L_{\text {self }}+L_{p s}\right)}\right) \sin (\omega t)
\end{gathered}
$$

where

$$
\omega=\left[\frac{1}{\left(L_{s e l f}+L_{p s}\right)\left(C_{e x t}+C_{s e l f}\right)}-\frac{R_{p s}{ }^{2}}{4\left(L_{s e l f}+L_{p s}\right)^{2}}\right]^{1 / 2}
$$

\section{Simulation MODEL}

A single-gap configuration was used in the simulation to investigate and validate the physics of the discharge process. The simulation region is shown in Fig. 3 with the geometry parameters listed in Table I. They were used in the previous experiments [27] and found to have a balanced discharge current and pulse duration. 


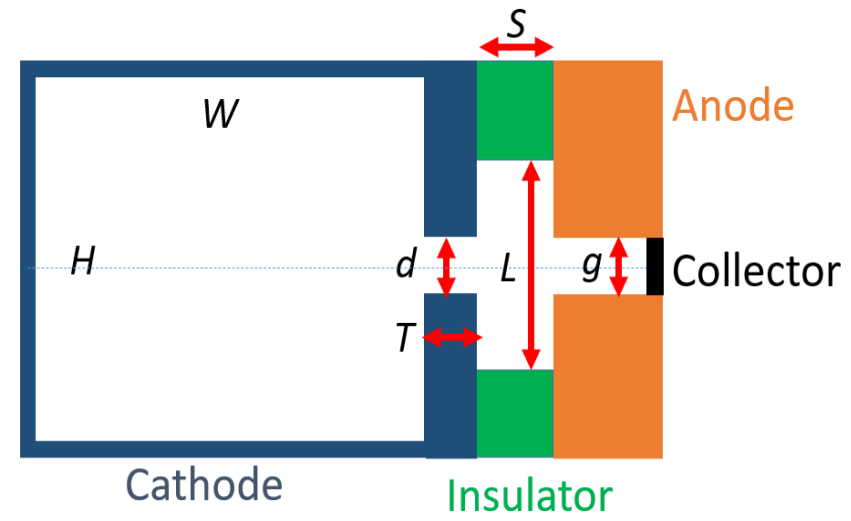

Fig. 3 2-D schematic view of the PS simulation model.

Table I the dimensions used in the experiments

\begin{tabular}{|l|l|}
\hline Cavity height $H$ & $50.0 \mathrm{~mm}$ \\
\hline Cavity Width $W$ & $50.0 \mathrm{~mm}$ \\
\hline Cavity aperture thickness $T$ & $3.0 \mathrm{~mm}$ \\
\hline Cavity aperture size $d$ & $3.0 \mathrm{~mm}$ \\
\hline Insulator thickness $S$ & $5.0 \mathrm{~mm}$ \\
\hline Insulator inner diameter $L$ & $10.0 \mathrm{~mm}$ \\
\hline Anode beam aperture $g$ & $3.0 \mathrm{~mm}$ \\
\hline
\end{tabular}

The PIC simulations of PS discharge combine multiple unique features, including large simulation volume, high plasma density which requires small mesh grids to satisfy the Courant criterion, and long simulation duration of the discharge process compared with the time step. It is not practical to simulate in $3 \mathrm{D}$ and hence the 2D open-sourced PIC code XOOPIC was used [18]. It is relatively easy to add new models and custom-defined diagnostics due to the Object-Oriented Architecture. The following physics processes were considered in the simulations.

(1) the elastic and ionizing collisions between the electrons and the background gas. In this paper, Argon was used. Three collision processes were implemented in XOOPIC, including the elastic collision $\left(e^{-}+A r \rightarrow A r+e^{-}\right)$, the excitation of upper molecular states $\left(e^{-}+A r \rightarrow A r^{*}+e^{-}\right)$, and the ionization collision $\left(e^{-}+A r \rightarrow A r^{+}+2 e^{-}\right)$. This process was simulated by the Monte Carlo algorithm using a cross-section model obtained from experimental data.

(2) To avoid the explosive increase of the particle numbers due to the ionization process. The particles are allowed to be combined as larger particles.

(3) The secondary electrons are produced when the electrons and the ions reach the cavity wall. Vaughan's equation and Furman's equation are two models commonly used to calculate the secondary electrons yield (SEY) [28, 29]. Vaughan's true SEY function $\delta$ is

$$
\delta / \delta_{0 m}=\left(v e^{1-v}\right)^{k}
$$

where $\delta_{0 m}$ is its maximum value. $v=\left(E_{0}-E_{\text {min }}\right) /\left(E_{0 m}-\right.$ $\left.E_{\text {min }}\right)$, where $E_{0}$ is the energy of the incident primary electron. $E_{\text {min }}$ is the minimum energy to generate the secondary electrons. A commonly used value is $12.5 \mathrm{eV} . E_{0 m}$ is the incident electron energy with the maximum SEY. The parameter $k$ at different $v$ values are

$$
\left\{\begin{array}{c}
k=k_{1}=0.56, \text { for } v<1 \\
k=k_{2}=0.25, \text { for } 1<v \leq 3.6 \\
\delta=1.125 \delta_{0 m} / v^{0.35}, \text { for } v>3.6
\end{array}\right.
$$

Vaughan's model also gives the angular dependent SEY,

$$
\left\{\begin{array}{l}
E_{0 m}(\theta)=E_{0 m}(0)\left(1+k_{s E} \theta^{2} / 2 \pi\right) \\
\delta_{0 m}(\theta)=\delta_{0 m}(0)\left(1+k_{s \delta} \theta^{2} / 2 \pi\right)
\end{array}\right.
$$

where $\theta(0 \leq \theta<\pi / 2)$ is the incident angle of the primary electrons. $\delta_{0 m}(0)$ is the maximum SEY normal to the surface. $k_{s E}$ and $k_{s \delta}$ are the smooth factors for $E_{0 m}(0)$ and $\delta_{0 m}(0)$, respectively, and normally 1.0 are used.

Furman's formula is based on a probability model and the experimental data. The SEY is

$$
\delta=\frac{s\left(E_{0} / E_{0 m}\right)}{s-1+\left(E_{0} / E_{0 m}\right)^{s}} \delta_{0 m}
$$

where $s(s>1)$ is a constant. The SEY with considering the incident angle $\theta\left(0 \leq \theta \leq 84^{\circ}\right)$ is

$$
\left\{\begin{array}{l}
E_{0 m}(\theta)=E_{0 m}(0)\left[1+t_{1}\left(1-\cos ^{t_{2}} \theta\right)\right] \\
\delta_{0 m}(\theta)=\delta_{0 m}(0)\left[1+t_{3}\left(1-\cos ^{t_{4}} \theta\right)\right]
\end{array}\right.
$$

$t_{1}, t_{2}, t_{3}$, and $t_{4}$ are material dependent coefficients that can be found in [28]. Some comparisons of the two models can be referred in [30]. Both models did not show significant differences in the simulations. The ion impact SEY is not considered since the ion has a much smaller velocity.

(4) The field emission electrons at the cathode and anode surface. The Fowler-Nordheim field emission model was used [31]. The work function for stainless steel that is used as the material of the discharge cavity was $4.3 \mathrm{eV}$.

(5) The external circuit model described in the previous section was added to the original code. As the $L_{p s}$ and $R_{p s}$ in Eq. 2 represent the statistical behavior of the discharge process. They are time-dependent and depend on different discharge conditions. It is not feasible to calculate these values for each time step of the PIC calculation. Therefore, a simplified circuit model was used. In a practical PS discharge experiment, a large $R_{\text {charge }}$ is normally used to reduce the impact on the charging power supply. Eq. 1 can therefore be rewritten as

$$
\left(C_{\text {ext }}+C_{\text {self }}\right) d V=-I d t=-d Q
$$

For each time step, the deposited charge at the anode was counted and used to update the voltage between the cathode and anode of the discharge chamber. The updated voltage then became the new boundary conditions to solve the Poisson equations for the electric field, which was used to advance the particle momentum.

In the simulation, the parameters of the external circuit are $R_{\text {charge }}=1 \mathrm{M} \Omega, C_{\text {ext }}=470 p F$, and the charging voltage $V_{c}=12 \mathrm{kV}$, respectively. The pressure of the background gas was 0.18 Torr, and the gas density was $5.8 \times 10^{21} \mathrm{~m}^{-3}$. The mesh grid was $50 \mu \mathrm{m}$ for both coordinates. The time step was chosen as $1 \times 10^{-13} \mathrm{~s}$ to satisfy the Courant-Friedrichs-Levy (VFL) condition. The numerical weight of the particles is $2 \times 10^{3}$. To avoid the exponential growth of the particle numbers due to the avalanche ionization process, the maximum number of each particle species, including the ionization electrons, field emission electrons, and Argon ions, was limited below $2 \times 10^{6}$. The particles with the same species were combined and the particle number halved if it exceeded the threshold. 


\section{Simulation Results}

The PS discharge normally operates either at the selfbreakdown or triggered mode. When operating at selfbreakdown mode, either the external charging voltage or the pressure inside the cavity is swept gradually to reach the breakdown point. The ignition of the breakdown process started with the free electrons in the background gas. The triggered mode is preferred in practical applications as a small jitter can be achieved. The breakdown can be triggered by applying an external trigged pulse on a sharp needle to create a seed electron source. Then the seed electrons will ionize the background gas and achieve avalanche breakdown. In the simulations, an electron beam pulse with an energy of $1 \mathrm{kV}$ and a duration of $2 \mathrm{~ns}$ injected from the left side of the discharge cavity was used as a seed source to trigger the discharge process. Fig. 4 shows the breakdown time as the function of the number of the injected seed electrons. The breakdown time is defined as a rapid increment of the discharge current. It corresponds to the starting of the super-dense glow discharge stage. An electron number of $10^{10}$ is required to ignite the breakdown at about 500 ns. A smaller value will result in a much longer and larger jitter in the breakdown time, as shown in the error bar of Fig. 4. A larger value will reduce the breakdown time down to $\sim 200 \mathrm{~ns}$. However, it is not significant if it is beyond $10^{11}$. This indicates the time needed to ionize the background gas and to form the virtual anode.

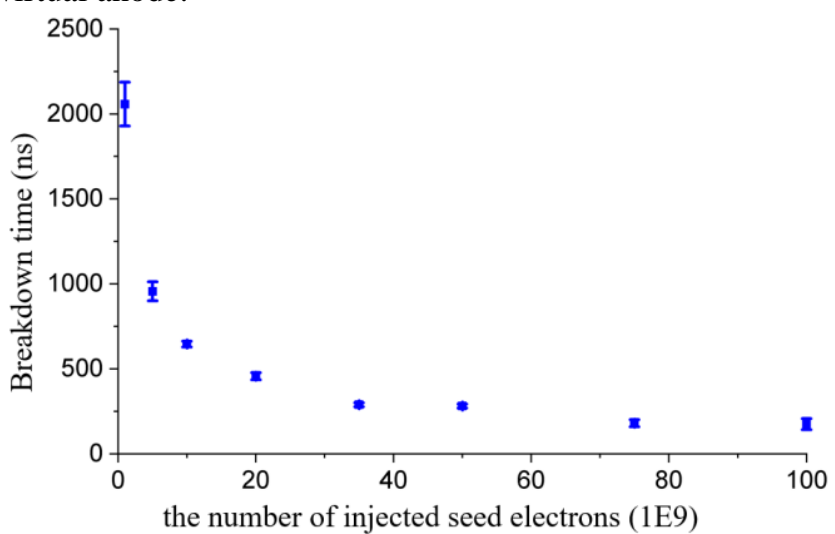

Fig. 4 breakdown time as the function of the number of injected seed electrons.

Typical plots of electron phase space and the contour lines of the potential at the different discharge stages are shown in Fig. 5 . The triggering electron source firstly ionizes the background gas. At this stage (Fig. 5(a)), the potential line across the cathode and anode have not yet penetrated the discharge cavity much. Therefore, only the electrons close to the aperture see the accelerating electric field. It is the pre-discharge discharge (Townsend discharge) phase and the current is small and grows slowly. As more and more electrons are extracted from the discharge cavity, the ions are leftover to form a positive space charge initially between the gap of the cathode and anode and then at the cathode aperture. The positive space charges deform the electric field caused by the potential between the cathode and anode and make them look like a part of the anode, which is a virtual anode [11]. This attracts the electrons to move to the aperture in this hollow cathode discharge stage, as shown in Fig. 5(b). As the density of the ions is sufficiently large, the equipotential line penetrates the cavity as shown in Fig. 5(c). Simulations show a relatively stable discharge current will stay for a few nanoseconds, then the virtual anode grows rapidly, a large number of electrons are generated due to the strong ionization of the background gas, which results in a rapid increase of the discharge current and initiation of the condense discharge glow process, as shown in Fig. 5(d).

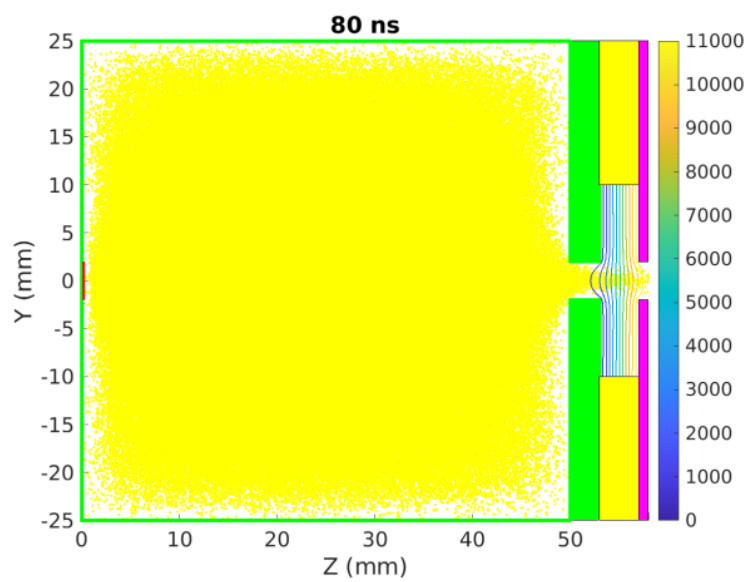

(a)

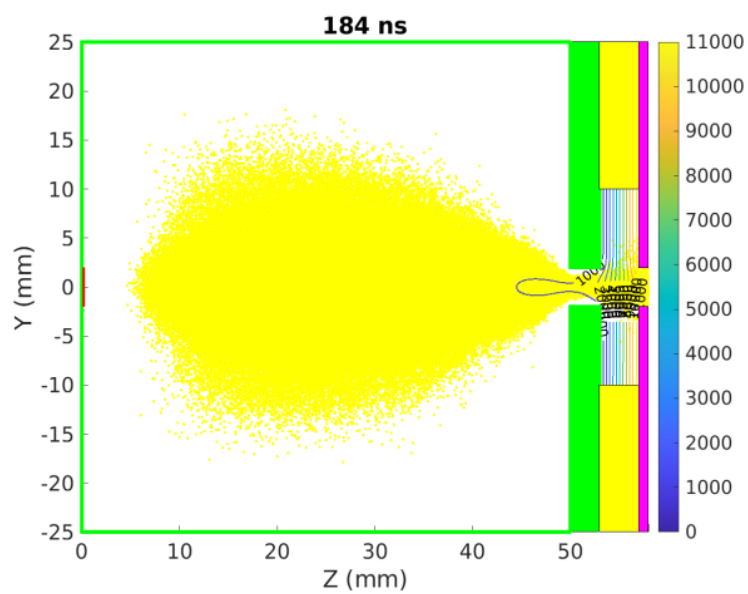

(b)

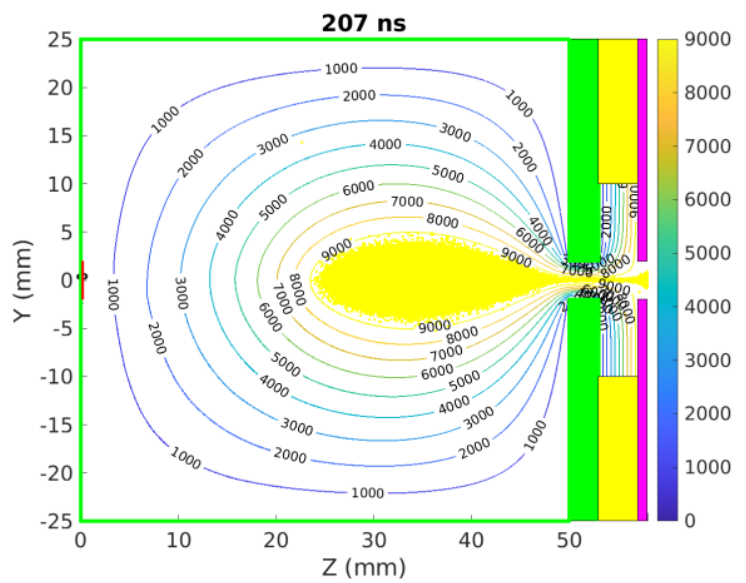

(c) 


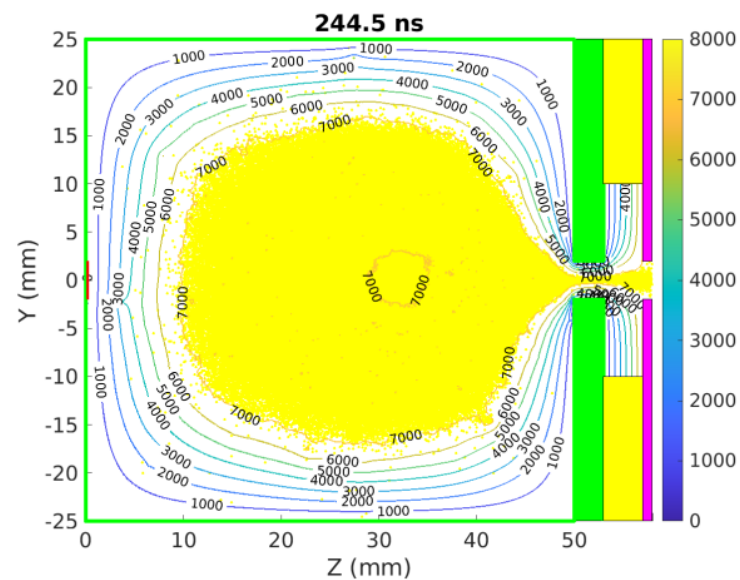

(d)

Fig. 5 The discharge processes at different times (a) $80 \mathrm{~ns}$, (b) $184 \mathrm{~ns}$, (c) $207 \mathrm{~ns}$, and (d) $244.5 \mathrm{~ns}$.

The discharge processes are similar for different geometry parameters and gas pressure, however, the discharge peak current and time are different, which have been presented in [15]. The general trends include: a larger cavity depth $W$ or cavity height $H$ will have a larger discharge peak current due to the larger gas volume; a narrower aperture size $d$ or a larger aperture depth $T$ will result in a larger discharge peak current and longer discharge time due to the slower penetration of the electric field; a higher charging voltage or a smaller insulation distance $S$ will result in a faster discharge process, however, it does not affect the peak discharge current significantly.

More discharge characteristics can be studied from the timevarying data, including the discharge voltage and current. The different discharge stages can be classified from the amplitudes of the discharge currents as well as the phase space of the electrons, as shown in Fig. 6. Both the simulation and measurement results have similar trends. The current is produced from the avalanching ionization of background gas due to the triggering electrons. The electrons close to the cavity aperture are then extracted to the anode due to the electric field between the cathode and the anode. If the aperture diameter is small or its thickness is large, the Townsend stage can be longer. As more and more electrons are extracted, more Argon ions are leftover. The electric field between the cathode and anode is gradually penetrated inside the cavity. It speeds up the ionization process and leads to a larger discharge current. The feature of the transition from the Townsend stage to the hollowcathode discharge stage is the formation of the virtual anode. When the virtual anode forms, most of the free electrons in the discharge cavity were extracted quickly and it results in the peak current at the end of the Townsend stage. The current in the hollow-cathode discharge stage is generated from the ionization of the gas as a result of the growth of the virtual anode. The duration of this stage is significant depending on how fast the virtual anode grows to the edge of the cavity, and normally is in a range of tens of nanoseconds.

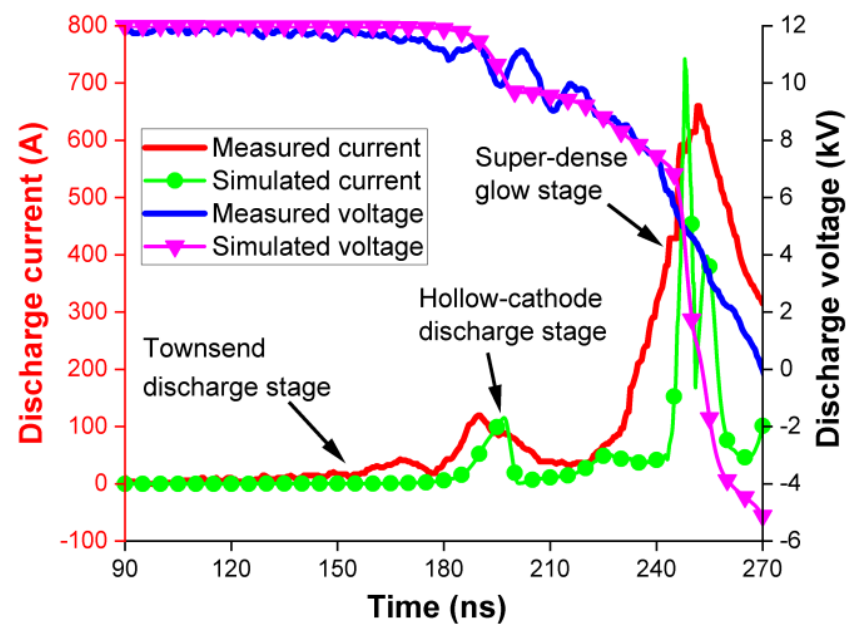

Fig. 6 The measured and simulated discharge currents and voltages.

Compared with the experimental results, the simulation shows the discharge current has a faster rise time and shorter pulse duration at the breakdown stage, which leads to a faster drop in the discharge voltage. It could be caused by the relatively slow response of the current diagnostics circuit (Rogowski coil) in the experiment.

It is interesting to know the composition of the current during the discharge process. From the simulations, the major electrons are generated from the ionization of the background gas. Although the field emission model is applied to the surface of the discharge cavity, there are no field emission electrons observed. The secondary electrons due to the collisions of the electrons and the ions with the metal surface were firstly generated at the cavity aperture, and then more were generated at the inner surface of the discharge cavity. Fig. 7 shows the peak density of the secondary electrons compared with the electrons generated from the ionization process. The density of electrons generated due to ionization was about three orders of magnitude higher than the secondary electrons. It indicated that secondary electrons created at the discharge cavity surface do not play a critical role during the discharge process.

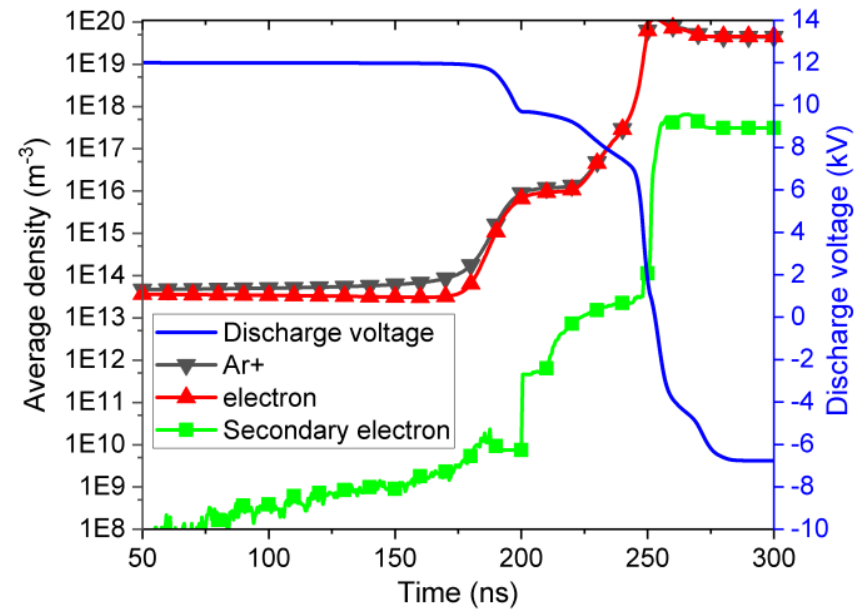

Fig. 7 The peak densities of the Argon ion, ionization electrons, and secondary electrons as the function of the discharge time.

One of the main application of the PS discharge is as the electron beam source that is extracted from the aperture of the 
anode. An aperture of $3.0 \mathrm{~mm}$ at the anode was set as a collector, as shown in Fig. 3, to diagnose the extracted electron beam. The electrons landing at the collector were captured and postprocessed. The energy of the extracted electrons was processed as the lower energy and higher energy regimes, which are divided into $10 \%$ segments of the charging potential $(1.2 \mathrm{keV}$ in this case). Fig. 8 shows the electron energy at maximum probability and its corresponding density as functions of the discharge time for both regimes. The error bar indicates the spread of the electron's energy. At the Townsend discharge stage, both low energy and high energy electrons had similar densities of about $2 \times 10^{13} \mathrm{~m}^{-3}$. The high-energy electrons were generated in the discharge cavity and then were accelerated by the electric field between the cathode and anode. The energy followed the trend of the discharge voltage and has a spread of around $10 \%$. The low-energy electrons had average energy of $500 \mathrm{eV}$. They are more likely generated close to the collector due to the ionization process.

At the hollow-cathode stage, the density of the electrons increased. As seen in Fig. 8, from 200 to 220 ns, only low energy electrons were observed. This corresponds to the time that the virtual anode forms and all the pre-ionized electrons have been extracted, and to the time that the virtual anode starts to grow. During this period, most of the electrons were generated at the gap between the cathode anode therefore they had relatively low energy. However, the density of the electrons kept increasing to about $1 \times 10^{18} \mathrm{~m}^{-3}$ due to the contribution of the electrons and ions to the ionization.

At the later stage of the hollow-cathode discharge, the virtual anode grew rapidly, and its potential increased. More gas in the discharge cavity was ionized and the electrons gained higher energy (with an average energy of $2 \mathrm{keV}$ and a spread of $20 \%$ ) and were extracted through the aperture. At the super-dense glow discharge stage, the electron density could reach up to $2 \times 10^{20} \mathrm{~m}^{-3}$. At this stage, the discharge current reached its maximum, followed by a significant amount of energy spread of around $40 \%$.

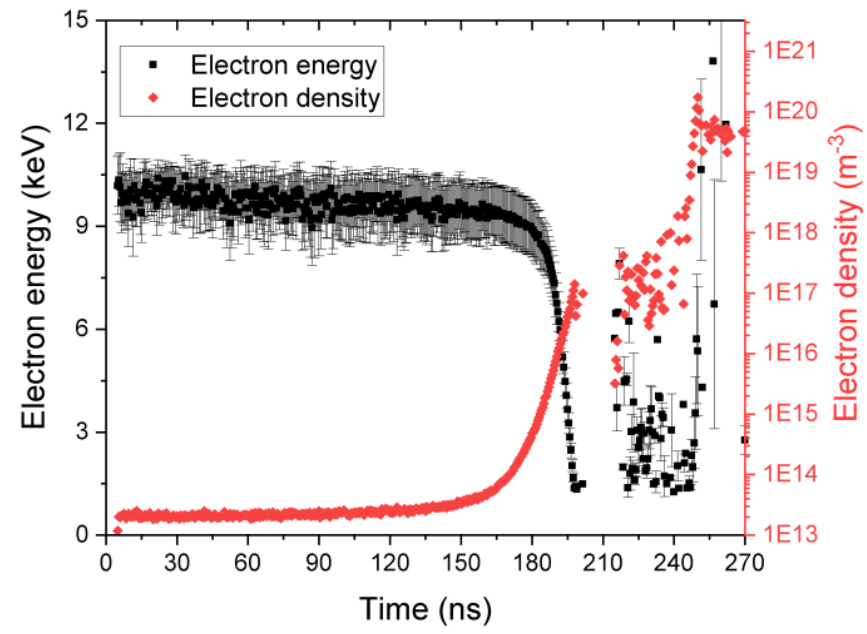

(a)

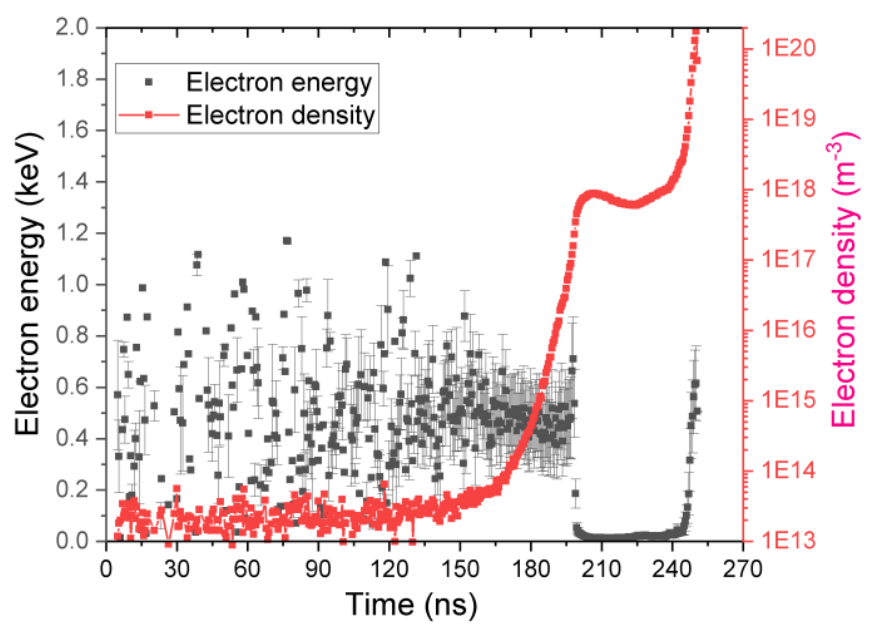

(b)

Fig. 8 The electron energy at maximum probability and its corresponding density as the function of the time, (a) high energy regime, (b) low energy regime.

\section{CONCLUSION}

The PS discharge is a dynamic process. The plasma density can change from $10^{13} \mathrm{~m}^{-3}$ to $10^{20} \mathrm{~m}^{-3}$ within $1 \mu \mathrm{s}$. In this paper, the PS discharge process including the external circuit was studied through particle-in-cell simulations. The composition of the electrons at different discharge stages was analyzed. The density of the secondary electrons produced at the cavity wall was about $1 \times 10^{13} \mathrm{~m}^{-3}$ at the hollow-cathode discharge stage, while the electrons generated from the ionization process have a density of $1 \times 10^{16} \mathrm{~m}^{-3}$, to make it the major source of the electrons.

The electron beam extracted from the discharge cavity at different discharge stages was also analyzed. The electron beams generated at the Townsend discharge, PS discharge and super-dense glow discharge stages have an energy spread of $10 \%, 20 \%$ and $40 \%$, respectively. The hollow-cathode discharge stage has balanced parameters between the beam energy, energy spread, and current density. It can be a better choice for applications such as driving the beam-wave interaction circuit to generate millimeter and terahertz waves. As the main source of the electrons is from the ionization of the background gas and not from the secondary electrons produced at the cavity wall, it will be useful to investigate different approaches to reduce the glow speed of the virtual anode and increase the duration of the hollow cathode discharge stage, therefore to increase the usable electron beam duration to improve the energy efficiency of the millimeter and terahertz devices.

\section{REFERENCES}

K. Frank and J. Christiansen, "The fundamentals of the pseudospark and its applications," IEEE Trans. Plasma Sci., vol. 17, no. 5, pp. 748-753, 1989, doi: 10.1109/27.41195.

J. Christiansen and C. Schultheiss, "Production of high current particle beams by low pressure spark discharges," Zeitschrift für Physik A Atoms and Nuclei, vol. 290, no. 1, pp. 35-41, 1979/03/01 1979, doi: 10.1007/BF01408477.

M. Stetter et al., "Investigation of the different discharge mechanisms in pseudospark discharges," IEEE Trans. Plasma Sci., vol. 23, no. 3, pp. 283-293, 1995, doi: 10.1109/27.402314. 
[4] S. Tricot, C. Boulmer-Leborgne, M. Nistor, E. Millon, and J. Perrière, "Dynamics of a pulsed-electron beam induced plasma: application to the growth of zinc oxide thin films," Journal of Physics D: Applied Physics, vol. 41, no. 17, p. 175205, 2008/08/12 2008, doi: 10.1088/0022-3727/41/17/175205.

[5] D. Bowes et al., "X-ray emission as a diagnostic from pseudosparksourced electron beams," Nuclear Instruments \& Methods in Physics Research Section B-Beam Interactions with Materials and Atoms, vol. 335, pp. 74-77, Sep 2014, doi: 10.1016/j.nimb.2014.06.008.

[6] C. S. Wong, H. J. Woo, and S. L. Yap, "A low energy tunable pulsed $\mathrm{X}$-ray source based on the pseudospark electron beam," Laser and Particle Beams, vol. 25, no. 3, pp. 497-502, Sep 2007, doi: $10.1017 / \mathrm{s} 0263034607000614$.

[7] W. He et al., "Generation of broadband terahertz radiation using a backward wave oscillator and pseudospark-sourced electron beam," Appl. Phys. Lett., vol. 107, no. 13, p. 133501, 2015/09/28 2015, doi: 10.1063/1.4932099.

[8] N. Kumar, R. P. Lamba, A. M. Hossain, U. N. Pal, A. D. R. Phelps, and R. Prakash, "A tapered multi-gap multi-aperture pseudosparksourced electron gun based X-band slow wave oscillator," Appl. Phys. Lett., vol. 111, no. 21, p. 213502, 2017/11/20 2017, doi: $10.1063 / 1.5004227$.

[9] K. Frank et al., "High-power pseudospark and BLT switches," IEEE Trans. Plasma Sci., vol. 16, no. 2, pp. 317-323, 1988, doi: 10.1109/27.3831.

[10] C. Jiang, A. Kuthi, and M. A. Gundersen, "Toward ultracompact pseudospark switches," Appl. Phys. Lett., vol. 86, no. 2, p. 024105, 2005/01/10 2005, doi: 10.1063/1.1852080.

[11] M. Zambra, J. Moreno, J. Inostroza, and J. C. Araneda, "Experimental observations of the virtual anode motion and streamer breakdown mechanisms in a pseudospark discharge," IEEE Trans. Plasma Sci., vol. 32, no. 1, pp. 221-226, 2004, doi: 10.1109/TPS.2004.823995.

[12] H. Taguchi et al., "Effects of Electrode Geometry on Breakdown Voltage of a Single-Gap Pseudospark Discharge," Jpn. J. Appl. Phys., vol. 37, no. Part 1, No. 1, pp. 303-307, 1998/01/15 1998, doi: 10.1143/jjap.37.303.

[13] M. Gastel, H. Hillmann, F. Muller, and J. Westheide, "Influence of the hollow cathode dimensions on the electron beam current in a pseudospark discharge," IEEE Trans. Plasma Sci., vol. 23, no. 3, pp. 248-253, 1995, doi: 10.1109/27.402309.

[14] J. Boeuf and L. C. Pitchford, "Pseudospark discharges via computer simulation," IEEE Trans. Plasma Sci., vol. 19, no. 2, pp. 286-296, 1991, doi: 10.1109/27.106826.

[15] S. O. Cetiner, P. Stoltz, P. Messmer, and J. L. Cambier, "Dependence of electron peak current on hollow cathode dimensions and seed electron energy in a pseudospark discharge," J. Appl. Phys., vol. 103, no. 2, p. 023304, 2008/01/15 2008, doi: 10.1063/1.2832507.

[16] Varun and U. N. Pal, "PIC Simulation to Analyze Peak Electron Current Generation in a Triggered Pseudospark Discharge-Based Plasma Cathode Electron Source," IEEE Trans. Electron Devices, vol. 65, no. 4, pp. 1542-1549, 2018, doi: 10.1109/TED.2018.2808175.

[17] X. Cao, J. Hu, R. Zhang, W. Huo, Y. Fu, and W. Zhao, "Dependence of pre-breakdown time on ionization processes in a pseudospark discharge," AIP Advances, vol. 7, no. 11, p. 115005, 2017/11/01 2017, doi: $10.1063 / 1.5003242$.

[18] J. P. Verboncoeur, A. B. Langdon, and N. T. Gladd, "An objectoriented electromagnetic PIC code," Computer Physics Communications, vol. 87, no. 1, pp. 199-211, 1995/05/02/1995, doi: https://doi.org/10.1016/0010-4655(94)00173-Y.

[19] T.-X. Corporation, "OOPIC Pro Object-Oriented Particle-in-Cell Simulation User's Manual," 2004.

[20] J. Hammel and J. Verboncoeur, "DC Discharge Studies Using PICMCC," 2003.

[21] V. Vahedi and M. Surendra, "A Monte Carlo collision model for the particle-in-cell method: applications to argon and oxygen discharges," Computer Physics Communications, vol. 87, no. 1, pp. 179-198, 1995/05/02/ 1995, doi: https://doi.org/10.1016/00104655(94)00171-W.

[22] V. Vahedi, G. DiPeso, C. K. Birdsall, M. A. Lieberman, and T. D. Rognlien, "Capacitive RF discharges modelled by particle-in-cell Monte Carlo simulation. I. Analysis of numerical techniques,"
Plasma Sources Science and Technology, vol. 2, no. 4, pp. 261-272, 1993/11/01 1993, doi: 10.1088/0963-0252/2/4/006.

[23] V. Vahedi, C. K. Birdsall, M. A. Lieberman, G. DiPeso, and T. D. Rognlien, "Verification of frequency scaling laws for capacitive radio - frequency discharges using two - dimensional simulations*," Physics of Fluids B: Plasma Physics, vol. 5, no. 7, pp. 2719-2729, 1993/07/01 1993, doi: 10.1063/1.860711.

[24] V. Vahedi and G. DiPeso, "Simultaneous Potential and Circuit Solution for Two-Dimensional Bounded Plasma Simulation Codes," J. Comput. Phys., vol. 131, no. 1, pp. 149-163, 1997/02/01/ 1997, doi: https://doi.org/10.1006/jcph.1996.5591.

[25] C. Lazzaroni and P. Chabert, "A global model of the self-pulsing regime of micro-hollow cathode discharges," J. Appl. Phys., vol. 111 , no. 5, p. 053305, 2012/03/01 2012, doi: 10.1063/1.3690943. H. J. Lee and J. K. Lee, "Time-Dependent Global-Model Simulation for a Pseudospark Discharge with a Cylindrical Hollow Cathode," Jpn. J. Appl. Phys., vol. 35, no. Part 1, No. 12A, pp. 6252-6258, 1996/12/15 1996, doi: 10.1143/jjap.35.6252.

[27] H. Yin, "Pseudospark Discharge and Cherenkov Maser Experiments," PhD, Department of Physics, University of Strathclyde, 1998.

[28] M. A. Furman and M. T. F. Pivi, "Probabilistic model for the simulation of secondary electron emission," Physical Review Special Topics - Accelerators and Beams, vol. 5, no. 12, p. 124404, 12/31/ 2002, doi: 10.1103/PhysRevSTAB.5.124404.

[29] R. M. Vaughan, "Secondary emission formulas," IEEE Trans. Electron Devices, vol. 40, no. 4, p. 830, 1993, doi: 10.1109/16.202798

[30] L. Zhang, W. L. He, A. W. Cross, A. D. R. Phelps, K. Ronald, and C. G. Whyte, "Numerical Optimization of a Multistage Depressed Collector With Secondary Electron Emission for an X-band GyroBWO," IEEE Trans. Plasma Sci., vol. 37, no. 12, pp. 2328-2334, Dec 2009, doi: 10.1109/tps.2009.2034164.

[31] R. H. Fowler and L. Nordheim, "Electron emission in intense electric fields," Proceedings of the Royal Society of London. Series A, Containing Papers of a Mathematical and Physical Character, vol. 119 , no. 781 , pp. $173-181,1928 / 05 / 01$ 1928, doi: 10.1098/rspa.1928.0091. 